\title{
The application of navigational risk analysis methods for designing pilot navigational systems
}

\author{
S. Gucma \\ Maritime University of Szczecin, Poland
}

\begin{abstract}
The article presents a method of estimating navigational safety in restricted areas using the method of navigational risk; a method of optimizing the designing of pilot navigational systems and prototypes of optimal pilot navigational systems (stationary and portable) constructed at the Maritime University.
\end{abstract}

\section{Introduction}

The admissible size of manoeuvring vessels is decreased and their movement parameters are altered in restricted areas at night and in times of impaired visibility. In the case of vessels operated on regular routes, hydrometeorological conditions cause them to be delayed, and attempts at eliminating those delays lead to heightened navigational risk. The restrictions on admissible size of manoeuvring vessels in these areas and their delays are caused by:

- $\quad$ inaccurate knowledge of the vessel manoeuvring in the area at each moment of performing the manoeuvre,

- $\quad$ inaccurate knowledge of the vessel's manoeuvring characteristics in particular navigational conditions.

These restrictions can be eliminated by applying specially designed pilot navigational systems.

The concept of a pilot navigational system consists in providing the master or the pilot conducting the vessel with information facilitating the safe performance of the planned manoeuvre in particular navigational conditions in set time. The information provided must be: 
- $\quad$ sufficient for the safe performance of the manoeuvre,

- $\quad$ presented in a form permitting the master or pilot to use it without being distracted from their running tasks bound with conducting the vessel.

The article presents a method of designing a pilot navigational system that fulfils the above conditions by:

- minimizing information indispensable for the safe performance of the planned manoeuvre in particular navigational conditions,

- a kind of visualising the minimal information amount enabling to master to translate it into the settings of the vessel's devices implementing the safe performance of the planned manoeuvre without being distracted from their running tasks bound with conducting the vessel.

- $\quad$ meeting the following criterion:

$$
R_{i j k x y} \leq R_{x y}^{d o p}
$$

where:

$$
\begin{aligned}
& R_{i j k x y}-\quad \begin{array}{l}
\text { navigational risk of performing the } j \text {-th manoeuvre by the } i \text {-th } \\
\text { vessel in } k \text {-th conditions in area (xy); }
\end{array} \\
& R_{l x y}^{d o p}-\quad \text { admissible navigational risk in area (xy). }
\end{aligned}
$$

\section{The assessment of navigational safety in restricted areas with the application of navigational risk analysis methods}

A vessel can manoeuvre safely only in an area where she fulfils at every point the condition of required depth. Such an area is called accessible navigational area, which can be presented in the form of area $\mathbf{D}$ of sets of point fulfilling the condition of required depth at moment $t$ [3].

The vessel performing a given manoeuvre in an accessible navigational area occupies a certain area determined by her successive locations in the area. The parameters of this area are random and depend on various factors. This area, calculated on a definite reliability level is called safe manoeuvring area. A safe manoeuvring area so defined can be presented in the form of area $\mathbf{d}_{\mathrm{ijk}}$ (set of points) and the basic navigational safety condition can be written down as follows [3]:

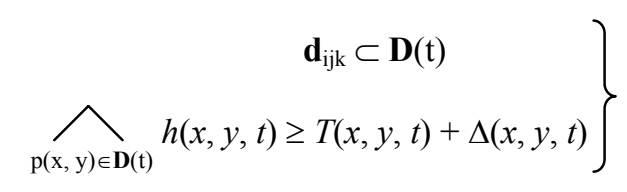

where:

$\mathbf{D}(t) \quad-\quad$ accessible navigational area (meeting the condition of accessible depth at moment $t$ ), 
$\mathbf{d}_{\mathrm{ijk}} \quad-$ accessible manoeuvring area (traffic lane) of the $i$-th vessel, performing the $j$-th manoeuvre in $k$-th navigational conditions,

$h(x, y, t)-$ the depth of the area at point with coordinates $(x, y)$ at moment $t$,

$T(x, y, t)-$ the draft of the vessel at area point with coordinates $(x, y)$ at moment $t$,

$\Delta(x, y, t)-\quad$ underkeel clearance at area point with coordinates $(x, y)$ at moment $t$.

Sets of points of the accessible navigational area $\mathbf{D}(t)$, as also the safe manoeuvring area $\mathbf{d}_{\mathrm{ijk}}$ can be identified with areas of definite linear parameters (Fig. 1).

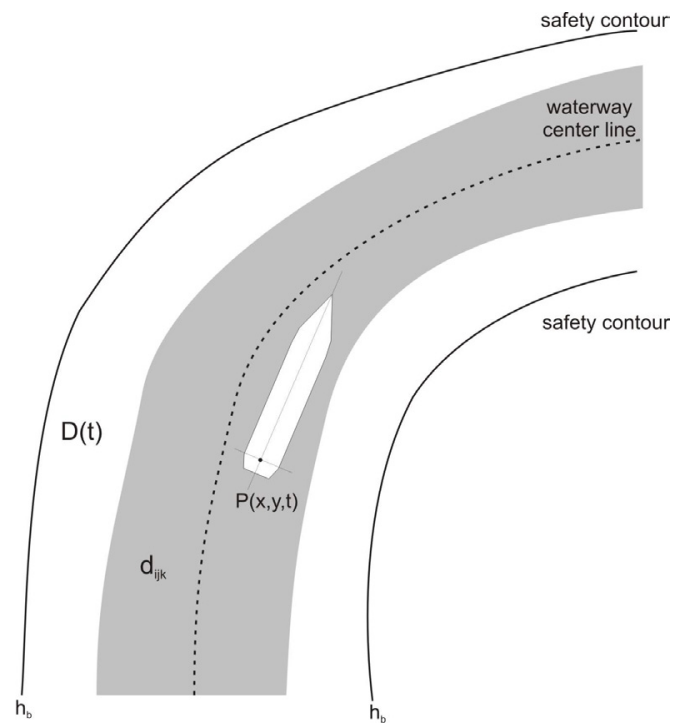

Figure 1: $\quad$ Sets of accessible navigational area $\mathbf{D}(t)$ and safe manoeuvring area $\mathbf{d}_{\mathrm{jk} \text {. }}$

Navigational safety in restricted areas is a concept which covers all problems bound with conducting the vessel from point A to point $\mathrm{B}$, fulfilling the basic navigational safety condition (2) throughout the route.

This safety can be presented in the form of the function:

$$
P_{i}=f\left(A_{i}, S_{i}, N_{i}, H_{i}, M_{i}, I_{i}, R_{i}\right)
$$

where:

$A_{i} \quad-\quad$ area parameters,

$S_{i} \quad-\quad$ vessel parameters,

$N_{i} \quad-\quad$ position fixing systems parameters,

$H_{i} \quad-\quad$ parameters of hydrometeorological conditions, 
$M_{i} \quad-\quad$ parameters of manoeuvre performed taking into consideration the human factor,

$I_{i} \quad-\quad$ traffic intensity parameters,

$R_{i} \quad-\quad$ traffic control system parameters.

The function of navigational safety is a dependent variable, conditioned by independent variables $A_{i}, S_{i}, N_{i}, H_{i}, M_{i}, I_{i}, R_{i}$, composed of a number of factors describing the system: vessel - area - position fixing system - prevalent hydrometeorological conditions - traffic intensity - traffic control system manoeuvring tactics.

In order to determine the effect of variables $A_{i}, S_{i}, N_{i}, H_{i}, M_{i}, I_{i}, R_{i}$ on navigational safety, it is necessary to assume a numerical index. This index defines the safety of navigation in restricted areas in a quantitative way. Currently research is conducted in the world aimed at making this index universal for all types of restricted areas and manoeuvres performed there. It is called navigational risk and the ability to estimate it will make it possible to assess unambiguously the safety of navigation in restricted areas.

Navigational risk is defined as the product of probability of failure occurrence and the consequences it can cause. Additionally, the definition of risk was supplemented by relative frequency of performing the manoeuvre under research. Assuming that failure and its results are independent occurrences, navigational risk can be presented in the form of product:

$$
R_{\mathrm{ijkxy}}=I_{\mathrm{ijkxy}} \cdot P_{\mathrm{ijkxy}}^{\mathrm{a}} \cdot S_{\mathrm{ijkxy}}
$$

where:

$I_{\mathrm{ijkxy}} \quad-$ average annual intensity (frequency) of performing the $j$-th manoeuvre by the $i$-th vessel in $k$-th navigational conditions in area (xy),

$P_{\mathrm{ijkxy}}^{\mathrm{a}} \quad-\quad$ probability of particular failure occurrence when performing the $j$ th manoeuvre by the $i$-th vessel in $k$-th navigational conditions in area (xy),

$S_{\mathrm{ijkxy}} \quad-\quad$ results caused by this failure when performing the $j$-th manoeuvre by the $i$-th vessel in $k$-th navigational conditions in area (xy).

At the same time it should be noted that the product $I_{\mathrm{ijkxy}} \cdot P_{\mathrm{ijkxy}}^{\mathrm{a}}$ is the probable number of a definite failure occurrence in a year, that is:

$$
a_{\mathrm{ijkxy}}=I_{\mathrm{ijkxy}} \cdot P_{\mathrm{ijkxy}}^{\mathrm{a}}
$$

The estimation of navigational risk consists in:

calculation of the probability of failure occurrence of a certain type when performing the $j$-th kind of manoeuvre in a set area restricted by the $i$-th kind of vessel in $k$-th navigational conditions, 
- $\quad$ estimating failure consequences of a certain type due to performing the $j$-th kind of manoeuvre in a set area restricted by the $i$-th kind of vessel in $k$-th navigational conditions.

Based on the definition of navigational risk the condition of safe definition of safe navigation has been determined:

$$
R_{\mathrm{ijkxy}} \leq R_{\mathrm{xy}}^{\mathrm{dop}}
$$

and after transformation:

$$
I_{\mathrm{ijkxy}} \cdot P_{\mathrm{ijkxy}}^{\mathrm{a}} \cdot S_{\mathrm{ijkxy}} \leq R_{\mathrm{xy}}^{\mathrm{dop}}
$$

\section{Designing pilot navigational systems when using navigational risk analysis methods}

Taking into consideration the tasks set for the PNS system and experiences concerning pilotage and the operation of similar systems in Poland and throughout the world, the assumptions for constructing a pilot navigational system can be presented as follows [2]:

1. The system is constructed especially for conducting navigation in restricted areas (pilotage navigation). It has features like:

- $\quad$ integration with the area,

- $\quad$ integration with the vessel,

- optimal information,

- optimal user interface,

- $\quad$ manoeuvring prediction and support.

2. The system is constructed in two versions:

- $\quad$ portable for pilots (small dimensions, autonomous power supply),

- $\quad$ stationary, for ferries and vessels continuously moving on particular restricted areas.

3. Beside standard imaging the system also has special kinds of imaging for performing particular manoeuvres in restricted areas, like:

- coastal imaging for the mooring manoeuvre,

- $\quad$ fairway imaging for the manoeuvres of fairway passage.

4. The information presented on indicators is optimal, determined on the basis of:

- $\quad$ expert research by pilots and ferry masters,

- $\quad$ simulation research conducted with pilots and ferry masters,

- $\quad$ real research conducted on sea ferries and vessels sailing in restricted areas.

Information indispensable for the safe performance of the planned manoeuvre can be divided into three types:

a. Information about the area, concerning safe isobaths of the nearest navigational obstacles and navigational marks $\left(\mathrm{L}_{1}\right)$. 
b. Information about the location of the waterline of the vessel in the area $\left(\mathrm{L}_{2}\right)$.

c. Information about the predicted trajectory of the vessel in the area, i.e. determining the future movement trajectory of the vessel's waterline in a definite time scale with current settings $\left(\mathrm{L}_{3}\right)$.

With a task set in this way and its restrictions, the function of purpose, which is information used for performing the planned manoeuvre in definite navigational conditions, will be minimized:

$$
Q_{X, Y}=\sum_{l=1}^{3} Q_{i j k l x y} \rightarrow \min
$$

with restrictions:

$$
\begin{gathered}
R_{i j k x y} \leq R_{x y}^{d o p} \\
Q_{i j k l x y} \leq Q_{l x y}^{d o p} \\
Z_{x y} \leq Z_{x y}^{a k c}
\end{gathered}
$$

where:

$Q_{x y} \quad-\quad$ information used in the researched system for the safe performance of manoeuvres in area $(x y)$;

$Q_{i j k 1 x y} \quad-\quad$ information concerning area $(x y)$ for the $i$-th vessel performing the $j$-th manoeuvre in $k$-th navigational conditions;

$Q_{i j k 2 x y} \quad-\quad$ information concerning the position of the $i$-th vessel in area $(x y)$ performing the $j$-th manoeuvre in $k$-th navigational conditions;

$Q_{i j k 2 x y}-$ information concerning prediction of the $i$-th vessel in area (xy)performing the $j$-th manoeuvre in $k$-th navigational conditions;

$R_{i j k x y} \quad-\quad$ navigational risk of performing the $j$-th manoeuvre by the $\mathrm{i}$-th vessel in $k$-th navigational conditions in area $(x y)$;

$R_{l x y}^{d o p} \quad-\quad$ admissible navigational risk in area $(x y)$;

$Q_{l x y}^{d o p} \quad-\quad$ maximal information amount of the $l$-th kind which the pilot or master can use in area $(x y)$;

$\mathrm{Z}_{\mathrm{xy}} \quad-\quad$ cost of building the system in area $(\mathrm{xy})$;

$Z_{x y}^{a k c} \quad-\quad$ acceptable cost of building the system in area (xy).

5. The system has an optimal user interface built especially for pilots and ferry masters. This interface was constructed on the basis of simulation research conducted with pilots and real research conducted on the Świnoujście Szczecin fairway. 
6. The system has a specially modified chart in its own format which has the indispensable elements for conducting pilotage navigation, e.g. the possibility of building an accurate, safe, dynamic isobath. It is also possible to use standard charts in accordance with IHO57, and also other systems of electronic charts.

7. Prediction of the manoeuvre performed will be applied in both the stationary and the portable system. In the stationary system manoeuvre prediction will be based on an accurate hydrodynamic model identified by 40-60 parameters of a given vessel. Prediction accuracy will be equal to $10 \%$ of the parameters of the performed manoeuvre (linear and temporal). In the portable system manoeuvre prediction will be based on the extrapolation of the vessel's current movement parameters determined on the basis of a positioning system in the vessel's general hydrodynamic model identified by $5-10$ parameters. Prediction accuracy will be equal to about $20 \%$ of the parameters of the performed manoeuvre (linear and temporal).

8. Manoeuvre supporting methods will be applied in the systems, e.g. the vessel's dynamic domains.

The basic research problems solved with the construction of the PNS can be formulated as follows:

1. Configuration of a suitable positioning subsystem fulfilling the requirements of:

- stationary PNS system,

- portable PNS system.

2. Construction of an electronic chart in a format adapted for conducting pilotage navigation.

3. Construction of an optimal information imaging system in the PNS.

4. Construction of an optimal user interface designed especially for pilots using the PNS.

5. Construction of a prediction system for:

- stationary PNS system,

- portable PNS system.

Research on optimizing information presented on the PNS indicator and the optimal solution for the user interface was conducted by means of a specially prepared three-stage optimization method. The method stages are:

Stage 1. Expert research.

Stage 2. Simulation research.

Stage 3. Real research.

The idea of the method prepared was the construction of a PNS prototype based on results obtained after the completion of each research stage. The prototype built was tested in the next research stage. After the third stage the research on the prototype was finished and the PNS entered the stage of designing and production. 
As a result of the completed second stage two PNS prototype came into being. These are:

1) stationary PNS, with a construction scheme presented in Fig. 2;

2) portable PNS, with a construction scheme presented in Fig. 3.

The basic parameters of these systems are given in Table 1 [1].

Currently these prototypes are verified in real conditions on the ferry $\mathrm{m} / \mathrm{f}$ "Jan Śniadecki" by means of methods described in the previous chapter.

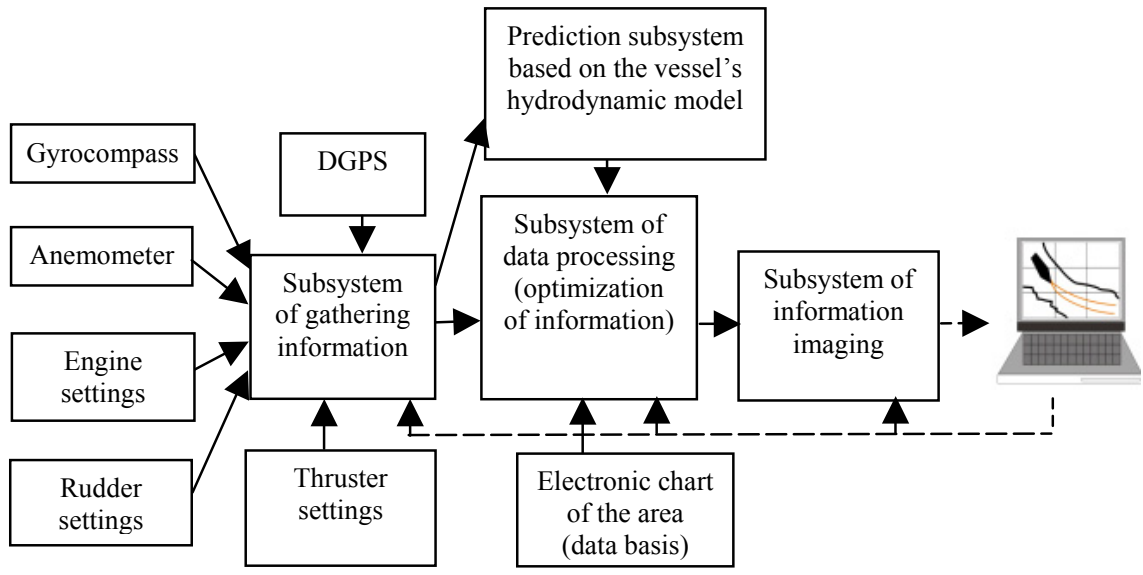

Figure 2: $\quad$ Scheme of stationary PNS.

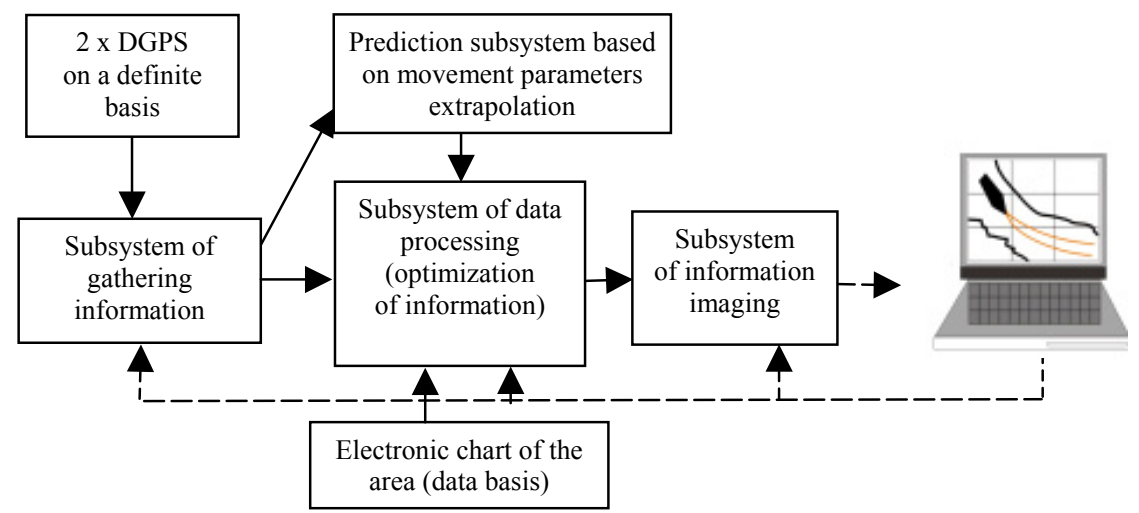

Figure 3: $\quad$ Scheme of portable PNS. 


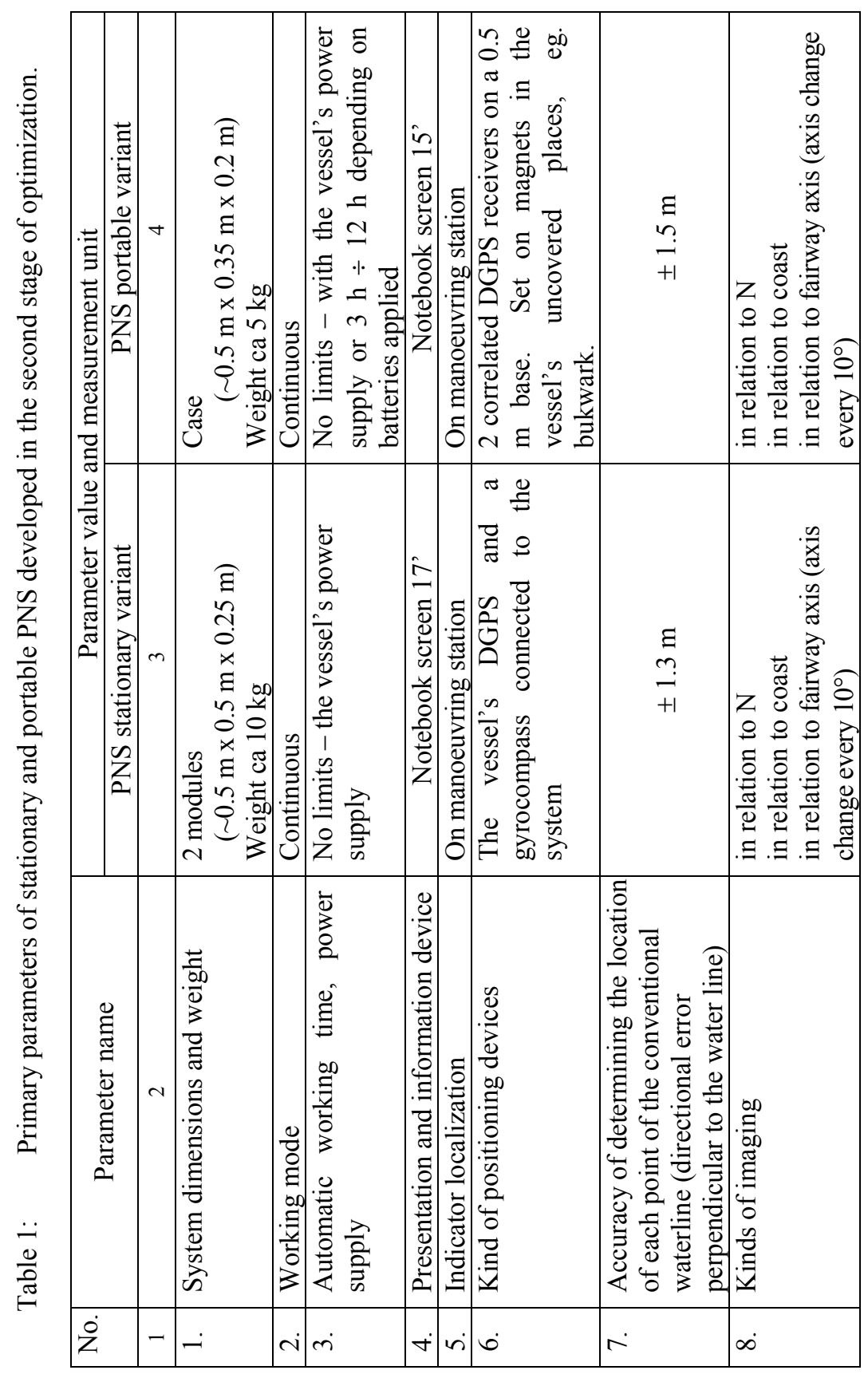




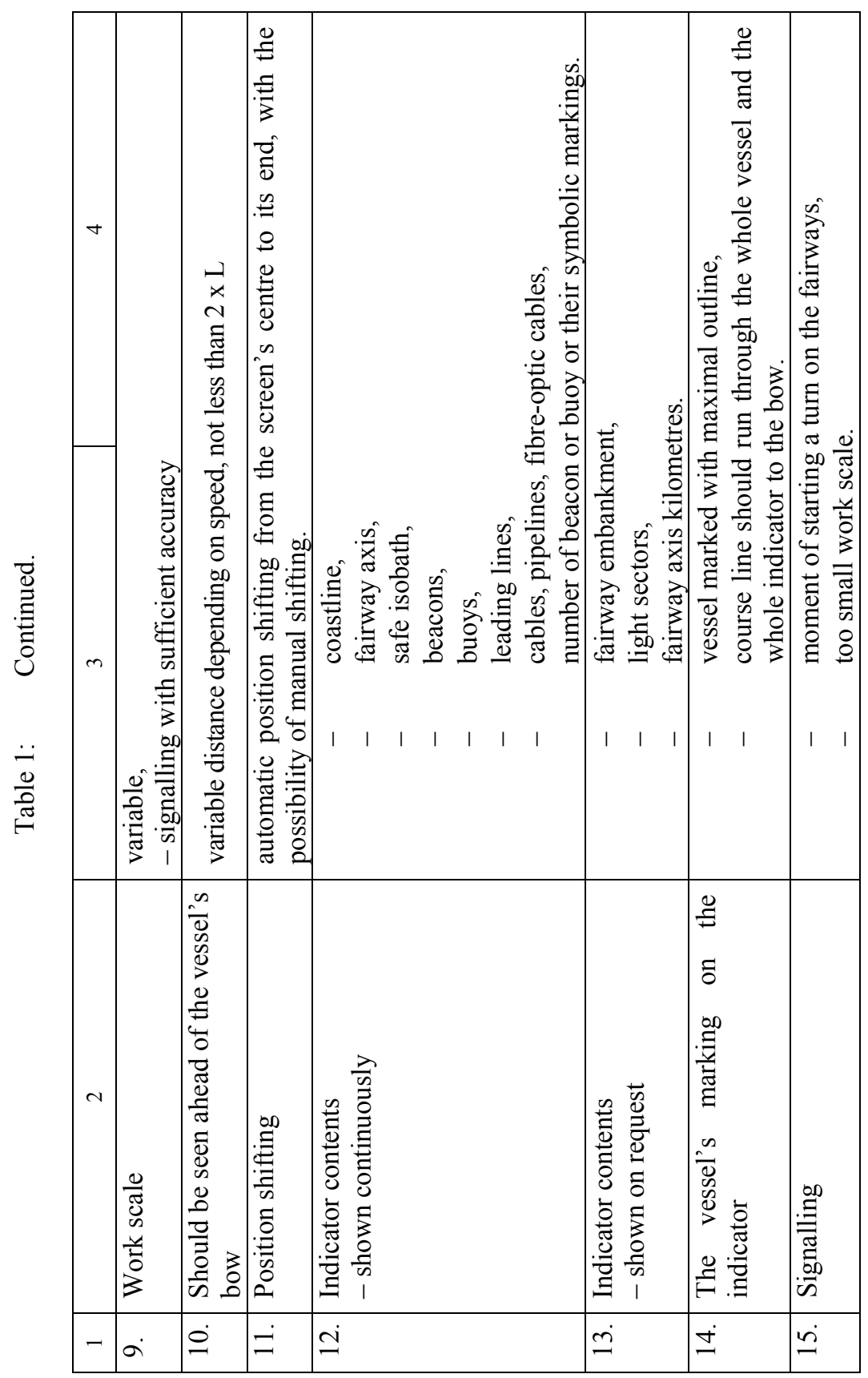




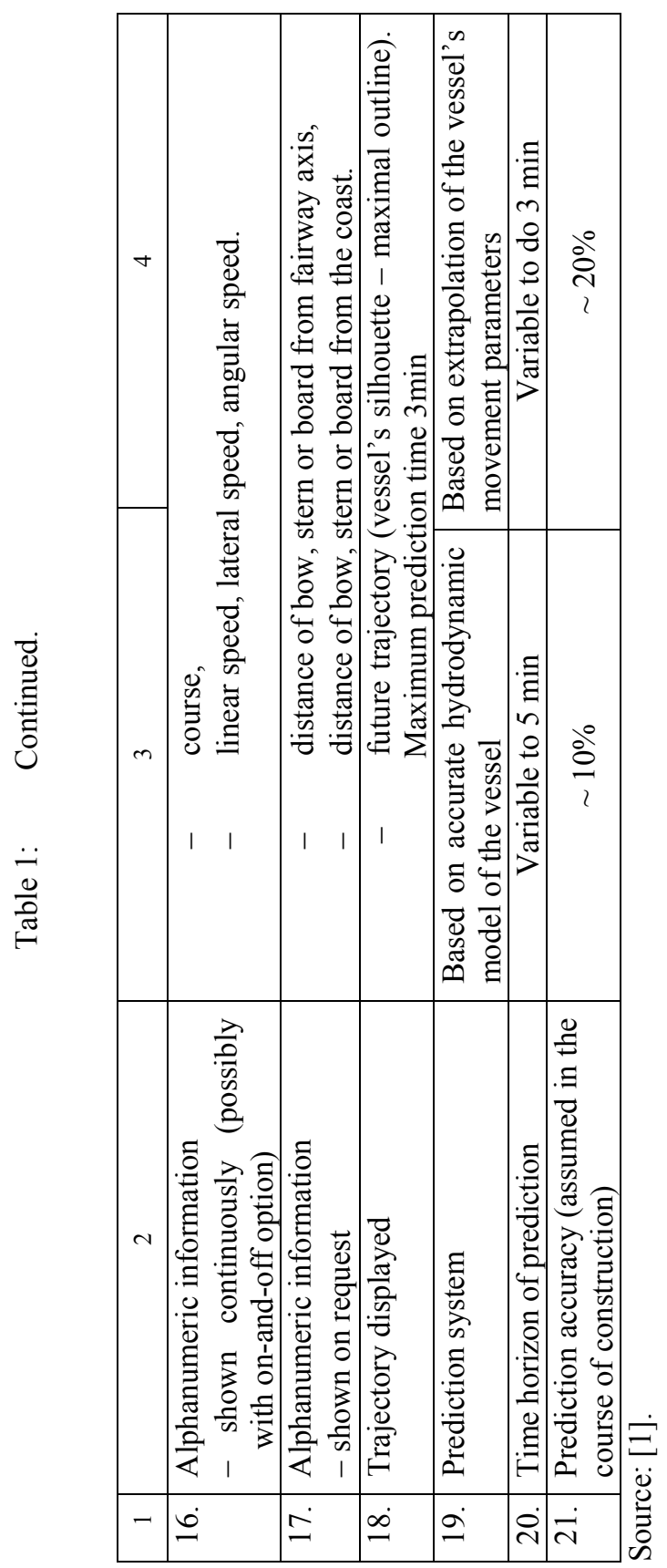




\section{Conclusions}

This article presents a method of designing a pilot navigation system, in which:

- $\quad$ the information indispensable for performing a planned manoeuvre in definite navigational conditions, with the condition of not exceeding the admissible risk fulfilled, is minimized;

- $\quad$ the designed system of visualizing this minimal information amount makes it possible for the pilot or the master to process it, without being distracted from the current tasks attendant on conducting the vessel.

This method has been used for the construction of prototypes of optimal stationary and portable navigation systems at the Maritime University of Szczecin. The parameters of these prototypes are presented in Table 1.

\section{References}

[1] Gucma M., Gucma S.: "Research results of prototypes of pilot navigation systems constructed at Maritime University of Szczecin". Explo-Ship, AM Szczecin 2006.

[2] Gucma S.: "An Optimal Solution to the Pilot Navigation System". XI Międzynarodowa Konferencja Inżynieria ruchu morskiego, ZN 6(78), AM Szczecin, 2005.

[3] Gucma S.: "Nawigacja pilotazowa". Fundacja Rozwoju Przemysłu Okretowego i Gospodarki Morskiej, Gdańsk 2004. 